\title{
The Progress and Clinical Application of Breast Cancer Organoids
}

\author{
Jin $\mathrm{Yu}$, Wei Huang \\ Department of Oncology, Affiliated Zhongshan Hospital of Dalian University, Dalian, China
}

Breast cancer is the malignant tumor with the highest incidence in women. Nowadays, the objects in vitro of models of this disease are mainly from breast cancer cell lines and patient-derived patient-derived xenograft (PDX). However, there is a significant gap between traditional cell lines and breast cancer solid tumors, meanwhiles, PDX is not highly consistent with patients due to different species. As a techonlogy, obtaining patient-derived tumor cells, combined with three-dimensional culture technology, adding cytokines that promotes the proliferation of breast cancer stem cells and inhibit their apoptosis, breast cancer organoids form a structure in vitro which is similar to tumor in the body. This model can not only study the occurrence and envolution of breast cancer, but is more prominent in clinical application. screening drugs by high-throughput, personalized treatment, textingtoxicity and immunotherapy. This article will review the breast cancer organoids, in evolution, source, culture system and clinical applications.

Keywords: Breast cancer, Tumor organoids, Cytokines, Clinical applications

\section{Introduction}

Breast cancer refers to the most common malignancy in women. Its incidence rate ranks first among female malignancies, and it has been elevating (1). With the rise in the new patients and the potential variations in the molecular phenotype and genotype of breast cancer, a more efficient culture system maintaining the biological characteristics of breast cancer in vivo is critical to clinical research and transformation. Traditional breast disease models consist of cells and PDX. The former is simple and easy to adjust culture conditions, however, there are various

Received: May 11, 2020, Revised: July 22, 2020,

Accepted: July 23, 2020, Published online: August 31, 2020

Correspondence to Jin $\mathrm{Yu}$

Department of Oncology, Affiliated Zhongshan Hospital of Dalian

University, No. 6 Jiefang Street, Dalian 116001, China

Tel: +86-18742591860, Fax: +86-0411-87650211

E-mail: 2947665040@qq.com

(c) This is an open-access article distributed under the terms of the Creative Commons Attribution Non-Commercial License (http://creativecommons.org/ licenses/by-nc/4.0/), which permits unrestricted non-commercial use, distribution, and reproduction in any medium, provided the original work is properly cited.

Copyright (c) 2020 by the Korean Society for Stem Cell Research cross-contamination problems, and the genetic information and tumor heterogeneity of patients are difficult to retain in the passage process; the latter can act as a gold standard for revelant tumor studies. To a certain extent, it can indicate the occurance and evolution of patients' tumors, whereas its time-consuming, low success rate, high cost and ethical issues. Thus, novel breast cancer research models should be developed essentially. Organoids exploit cells exhibiting different differentiation potentials (e.g., embryonic stem cells, pluripotent stem cells, and tumor cells, and other sources of cells; they are combined with in vitro three-dimensional culture technology and introduce extracellular matrix analogs and cytokines to maintain stem cell proliferation and differentiation, as well as other components. Thus, the cells grow in a culture system close to the microenvironment of the body, and a cell tissue structure is formed similar to the structure of the source organ in the body. As compared with in vitro culture models (e.g., conventional cell line culture, human xenograft, and simple biological materials), tissues formed by organoid culture systems can achieve relatively complex structures and functions, currently the ideal in vitro culture in vitro. system. Moreover, organoid culture requires less tissue and exhibits a high success rate and 
avoids ethical disputes and species differences.

Tumor-like organ culture refers to a cross-concept accompanying the development of organoids and oncology, namely, tumor cells form microstructures similar to the source tumor tissue structure and gene expression lineage under appropriate culturing conditions, which can reveal the biology of the source tumor tissue. It acts as an effective model to study tumors and high-throughput screening drugs in vitro. At present, tumors (e.g., colorectal cancer, pancreatic cancer, liver cancer, prostate cancer and breast cancer) have achieved relatively mature organoid culture systems (2-6). To be specific, the breast cancer culture system usually introduces various cell growth factors to the matrigel to simulate the in vivo microenvironment of breast cancer cells; thus, breast cancer stem cells can maintain their original differentiation ability and long-term subculture in vitro. It has become a feasible biological model for in vitro studies on the occurrence and development of breast cancer, and it has been extensively employed in individualized treatment of breast cancer patients.

\section{The Evolution of Breast Cancer Organoids}

Cells exhibit a three-dimensional growth pattern in the body. Under conventional two-dimensional planar culture conditions, the cells are gradually flattened, losing some of their biological characteristics in the body; Thus, a low degree of reduction of the results of in vitro experiments is caused. Three-dimensional cultivation technology has been progressively developed. In 1907, Wilson (7) demonstrated that mechanically dissociated sponge cells can be recombined to form a complete organism. In 1977, Emerman and Pitelka (8) initially inoculated cells obtained from mouse breast tissue on a three-dimensional matrix surface; Besides, they added insulin, hydrocortisone and prolactin to differentiate isolated breast cells into acinar structures and the source. The tissues were compared and reported to exhibit a similar structure to that of mammary acinus, which was a precedent for "breast organoid" culture. In 1992, Petersen et al. (9) initially termed the use of breast cells to form microstructures as "organoids". With the advancement of stem cell biology, people have come to realize that breast cancer stem cells are the key to form organoids. Therefore, some researchers have attempted to add transforming growth factor $\alpha$ (TGF- $\alpha$ ) and fibroblast growth factor 7 (FGF7) to the medium to activate the MAPK signaling pathway on the breast, promote the proliferation and differentiation of breast cancer stem cells, sa well as forming a breast branch phenotype (10). Subsequently, Sato et al. (11) added Wnt and R-Sponding1, which induced the proliferation of Lgr5 + stem cells and stimulated $\beta$-catenin and Tcf4 to be up-regulated, as a result, the stem cell activity of the tumor cells was activated, as well as in vitro intestinal organoid culture. In 2012, de Visser et al. (12) reported that breast Lgr5 + cells as breast stem cells can long exist during the passage; under the action of cytokines, breast stem cells were found to be arranged to form a normal duct structure similar to breast. According to this theory, Sachs et al. (13) added the Neuregulin1 nuclear factor that facilitates self-proliferation and differentiation of breast cancer stem cells, as well as small molecule inhibitors A83-01 and SB202190 that inhibit apoptosis of breast cancer stem cells based on the original organ culture program. Therefore, the synthetic medium for breast cancer organoids was used to develop the system. These researchers cultivated 95 breast cancer organoids from 155 breast cancer surgical tissues and successfully established the first breast cancer organoid bank. The breast cancer-like organoids in the library cover a range of pathological types, molecular phenotypes and genotypes of breast cancer, which were adopted for high-throughput screening of drugs and editing of mutated genes for precision medical purposes. Over the past few years, with the optimization of digestion steps and the continuous improvement of experimental techniques (e.g., screening of breast cancer stem cells), the success rate of breast cancer organoid culture has been elevated to $87.5 \%$, arousing a wave of study and application of breast cancer organoids (Fig. 1) (14).

\section{Establishment of Breast Organoid Culture System}

Breast organoids act as vital branches of tumor organoids, whereas the construction of the culture system has long been explored and optimized to form an organoid culture system that exhibits common characteristics with other tumor organoids as well as targets breast cancer cell characteristics. The construction of breast cancer organoids primarily consists of three elements (i.e., breast cancer cells, stroma and cytokines).

\section{Cell sources of breast cancer organoids}

Immortalized cell lines: Applying standard cell lines can efficiently cultivate breast cancer organoids, continuously domesticate and enhance the stemness of breast cancer stem cells, as well as elevating the success rate of organoid cultures during the passaging process. The cell lines were tubular subtype MCF7 and basal subtype MDAMB-231; both cell lines were in organoid medium (e.g., 


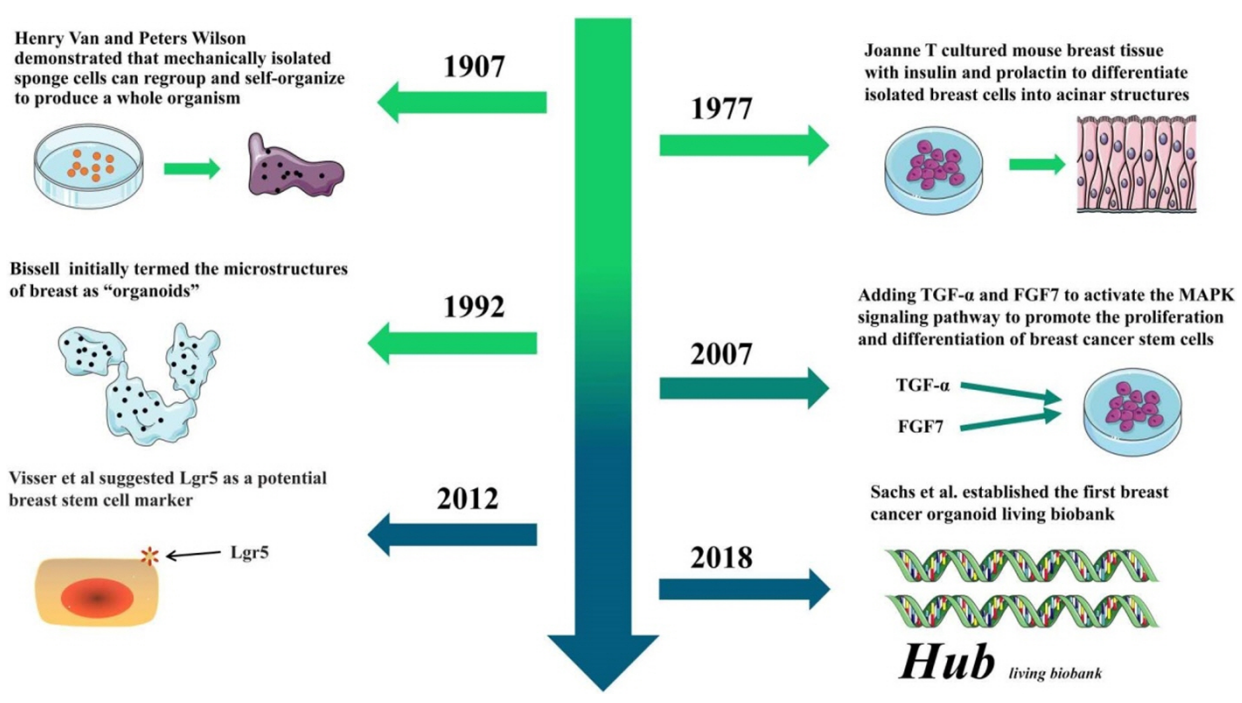

Fig. 1. The evolution of breast cancer organoids.
B27, EGF, and bFGF growth factors), and breast cancer organoids were formed one week later. After digestion and re-passaging of the seed plate, the proportion of organoids was elevated significantly, MCF7 increased from $8 \%$ to $33 \%$ in the third generation, MDA-MB-231 increased from $5 \%$ to $22 \%$ in the third generation, and the stem cell activity of breast cancer cells significantly increased $(15,16)$. With standard immortalized cell lines to form tumor-like organoids, breast cancer tumor-like organoids can be obtained efficiently. Cell lines with different phenotypes can reflect the biological characteristics of a range of breast cancer subtypes, which is an ideal tool for rapid initial screening of clinical drugs.

Cells of breast cancer tissue: Puncture and surgical tissue are more frequently employed. Compared with immortalized cells, breast cancer cells of this origin exhibit the advantage of being individualized. The breast cancer organs formed by breast cancer tissues from various patients are not limited by the single phenotype and genotype of the cell line. They can maximally restore the biological characteristics of tumors in patients in vitro. Sachs et al. (13) adopted surgical resection to acquire breast cancer tissues from a range of patients; the organoids formed after culture highly complied with the source tissues in pathological typing. Different clinical drug experiments were performed on these organoids to screen out sensitive drugs for different patients. Thus, it was found that the plan effectively enhances the treatment effect of patients. When the tumor sample contains over $5 \%$ of tumor stem cells, the organoid culture achieves the efficiency of $100 \%$, and the organoid of function express the diagnostic biomarkers of the corresponding tissues of patients (5).

This series of characteristics can act as an indicator of non-invasive monitoring of drug sensitivity variations in individual patients.

Circulating tumor cells (CTC): These cells refer to tumor cells spreading through the blood. usually composed of tumor cells that exhibit high invasive capacity and stem cell activity. Thus, the collection of the mentioned cells to form breast cancer organoids can indicate the invasion and metastasis characteristics of tumors in vivo. The study on specific surface markers and special signaling pathways and targeted killing of the mentioned tumor cells help effectively inhibit tumor recurrence and metastasis. Yu et al. (17) collected breast cancer cells in the blood of breast cancer patients. Besides, they performed pathological characterization and genome sequencing on breast cancer organs formed in vitro, as well as targeted gene mutation expression profiles of breast cancer organs from different patients. For the treatment, PI3K $\alpha$ inhibitor BYL719 and mTOR inhibitor everolis were employed., as well as other 12 diverse drug regimens. These researchers highlighted that according to the differences in the pathological characteristics of organoids of different mutant genes, different drug regimens can be taken for patients. Moreover, breast cancer organoids also showed different phenotypic variations for the differences in drug sensitivity. This series of characteristics can act as an indicator of non-invasive monitoring of drug sensitivity variations in individual patients.

Source of metastatic pleural effusion: Breast cancer patients do not find the lesion in time, probably due to untimely treatment, which often causes cancer cells to spread to the pleura and cause lymphatic obstruction, thus, reabsorption disorders are induced. Thus, breast cancer cells will appear in pleural fluid. Grimshaw et al. (18) 
centrifuged and enriched breast cancer cells in pleural fluid. In the medium supplemented with insulin, hydrocortisone, B27, and epidermal growth factor, breast cancer cells can form spherical structures. Besides, structural inoculation into immune-deficient mice can form breast tumors. Such discovery initially acquired breast cancer organoid cells with the pleural fluid pathway, besides, itprovided novel insights into the characteristics of breast cancer recurrence and metastasis in vitro and optimized the treatment plan.

Co-culture of mixed cells: The tumor microenvironment consists of tumor cells, tumor-related fibroblasts, endothelial cells, and immune inflammatory cells. Besides, breast cancer organs formed by single breast cancer cells cannot reproduce breast cancer in vivo. Accordingly, adding mesenchymal cells to the breast cancer organoid culture system can more significantly simulate the interaction between tumor cells and mesenchymal cells in vitro. In the process of breast cancer, the interaction between the two causes the reduction of local repair ability of breast cells, which is more consistent with the occurrence and development of solid tumors in vivo (19). Common mesenchymal cells include fibroblasts, immune cells, and myoepithelial cells. Breast branch epithelium and surrounding mesenchymal cells jointly regulate the development of the mammary gland and critically maintain the balance of the mammary gland. If the imbalance causes breast cancer, a co-culture model of breast cancer organoids and fi- broblasts can more effectively analyze the interaction between breast fibroblasts and epithelial organoids (20). Breast cancer organoids formed with BT-20 were co-cultured with macrophages, which achieved the interaction between the organoids and immune cells. Moreover, it could be observed under the microscope that this culture mode facilitates the hydrolysis of macrophages to the surrounding collagen (21).

\section{Three-dimensional culture substrate}

The matrix forming and maintaining the growth of organoids is a critical substance that mimics the microenvironment in the body. It creates attachment points for cells to support it, while providing nutrients to maintain cell growth. The common types include rat tail collagen, collagen and matrigel. Rat tail collagen is the earliest used matrix, which is easy to obtain and can support higher and cell survival rates. Since then, collagen and other matrices have been extensively used.; Among them, collagen IV, the major component of tumor stroma and laminin, is isolated from sarcoma and forms the main component of matrigel, which refers to the most used commercial matrix gel. Together with various growth factors, it forms a matrix protein mixture, which can more effectively replace the extracellular matrix and promote the growth, differentiation and tissue formation of breast cancer cells (22).

Table 1. The cytokines of cultural system and recommend concentration

\begin{tabular}{|c|c|c|}
\hline Cytokines & Function & Concentration \\
\hline $\begin{array}{l}\text { Epidermal } \\
\text { Growth Factor, } \\
\text { EGF }\end{array}$ & $\begin{array}{l}\text { The ligand of epidermal growth factor receptor (EGFR) promotes the proliferation of breast cancer } \\
\text { epithelial cells by promoting cell mitosis, to achieve the purpose of maintaining organoid growth } \\
\text { for a long time }\end{array}$ & $0.1 \sim 10 \mathrm{ng} / \mathrm{ml}$ \\
\hline $\begin{array}{l}\text { Fibroblast } \\
\text { Growth Factor7, } \\
\text { FGF7 }\end{array}$ & $\begin{array}{l}\text { Promoting the growth of epithelial cells; Improving the ability of breast cancer cells invades and } \\
\text { metastasizes; working with Wnt3a to promote the formation of spheroid structures in the } \\
\text { directionally differentiated endoderm and further expand the formation of breast cancer organoid }\end{array}$ & $50 \mathrm{ng} / \mathrm{ml}$ \\
\hline Wnt3a & $\begin{array}{l}\text { Lipoprotein receptor-related protein (LPR) ligand, which mainly regulates tumor cell proliferation } \\
\text { and differentiation }\end{array}$ & $50 \sim 500 \mathrm{ng}$ \\
\hline Noggin & $\begin{array}{l}\text { Bone morphogenetic protein (BMP) inhibitors, which can replace the missing Niche-related } \\
\text { signaling molecules to promote stem cell proliferation, increase the number of initial organoid } \\
\text { formation, and accelerate the rate of expansion }\end{array}$ & $100 \mathrm{ng} / \mathrm{ml}$ \\
\hline R-Spondin-1 & $\begin{array}{l}\text { G protein-coupled receptor } 4 / 5 \text { (LGR4 / 5) ligand, a Wnt pathway agonist, which riches in leucine } \\
\text { repeats, can maintain stem cell population growth for a long time in vitro }\end{array}$ & $500 \mathrm{ng} / \mathrm{ml}$ \\
\hline Neuregulin1 & $\begin{array}{l}\text { Neuregulin1:EGF receptor tyrosine kinase } 3 \text { and } 4 \text { (ERTK3 / 4) ligands are involved in breast } \\
\text { formation and tumorigenesis }\end{array}$ & $100 \mathrm{~g} / \mathrm{l}$ \\
\hline Y-27632 & $\begin{array}{l}\text { Y-27632:Rho-related coiled-coil formation protein kinase (ROCK) inhibitors can effectively reduce } \\
\text { the apoptosis of stem cells during the process of culture and improve the state of the medium }\end{array}$ & $10 \mathrm{mM}$ \\
\hline A83-01 & $\begin{array}{l}\text { Tumor growth factor- } \beta 1 \text { (TGF- } \beta \text { 1) inhibitor that inhibits the differentiation of pluripotent stem } \\
\text { cells, maintains self-renewal and improves expansion efficiency }\end{array}$ & $1 \mu \mathrm{M}$ \\
\hline SB202190 & MAPK inhibitor that promotes the stability of pluripotent stem cells & $1 \mu \mathrm{M}$ \\
\hline
\end{tabular}




\section{Cytokines and functions}

To achieve long-term in vitro culture of breast cancer organoids, multiple growth factors should be introduced to form a supporting culture system that meets the needs of a range of formation stages of organoids. Most of them cover growth factors associated with breast cancer stem cell proliferation and differentiation and inhibit breast cancer stem cell apoptosis, a small molecule inhibitor that maintains long-term in vitro culture of organoids consists of two parts. R-sponding, Wnt and EGF are critical to breast cancer cell localization, attachment and activation of signaling pathways, termed as the "troika for organoid culture" $(23,24)$. According to the biological role and molecular matrix of cytokines, they can be roughly split into the following categories (Table 1 and Fig. 2) (10, 19, 24-27).

Promote breast cancer cell proliferation and differentiation: Epidemical Growth Factor (EGF) can effectively facilitate the proliferation and division of various epithelial cells. Dontu et al. (25) initially added EGF to breast cancer culture medium. After 15-day culture, in contrast to the blank group, it was found that the treatment group can induce the formation of mammary tubules and increase breast cancer organs by nearly 30 times, i.e., the rate of organoid formation can be elevated; Noggin and R-Spondin-1 can also elevate the number of organoid formation and the rate of organoid formation.

Regulate the Wnt signaling pathway: Wnt-3a binds the Wnt ligand secreted by breast cancer stem cells to the corresponding receptor; subsequently, it regulates such signaling pathway, facilitates the proliferation of breast cancer stem cells and maintains the tumor stem cell subpopulation with R-Spondin-1 Long-term in vitro culture (26).

Molecular inhibitors: Including A83-01, Y-27632 and SB202190, A83-01 is an ALK4 / 5/7 inhibitor, promoting tumor cell proliferation (13). The early application of Y27632 primarily aims to avoid the "Nest apoptosis" of tumor cells after ex vivo; it helps tumor cells split and proliferate in vitro, inhibits the apoptosis of breast cancer stem cells and maintains long-term in vitro culture of stem cells with proliferative and differentiation capabilities. SB202190 is a p38 inhibitor, which is not conducive to the formation of organoids when the concentration exceeds $1 \mu \mathrm{M}$ (27-30).

\section{Application of Breast Cancer Organoids}

Organoid functions effectively reflect tumor heterogeneity, and gene sequencing of organoids formed in culture reveals that most organoid functions can display genes of source tissues well, even after prolonged passage, orga-

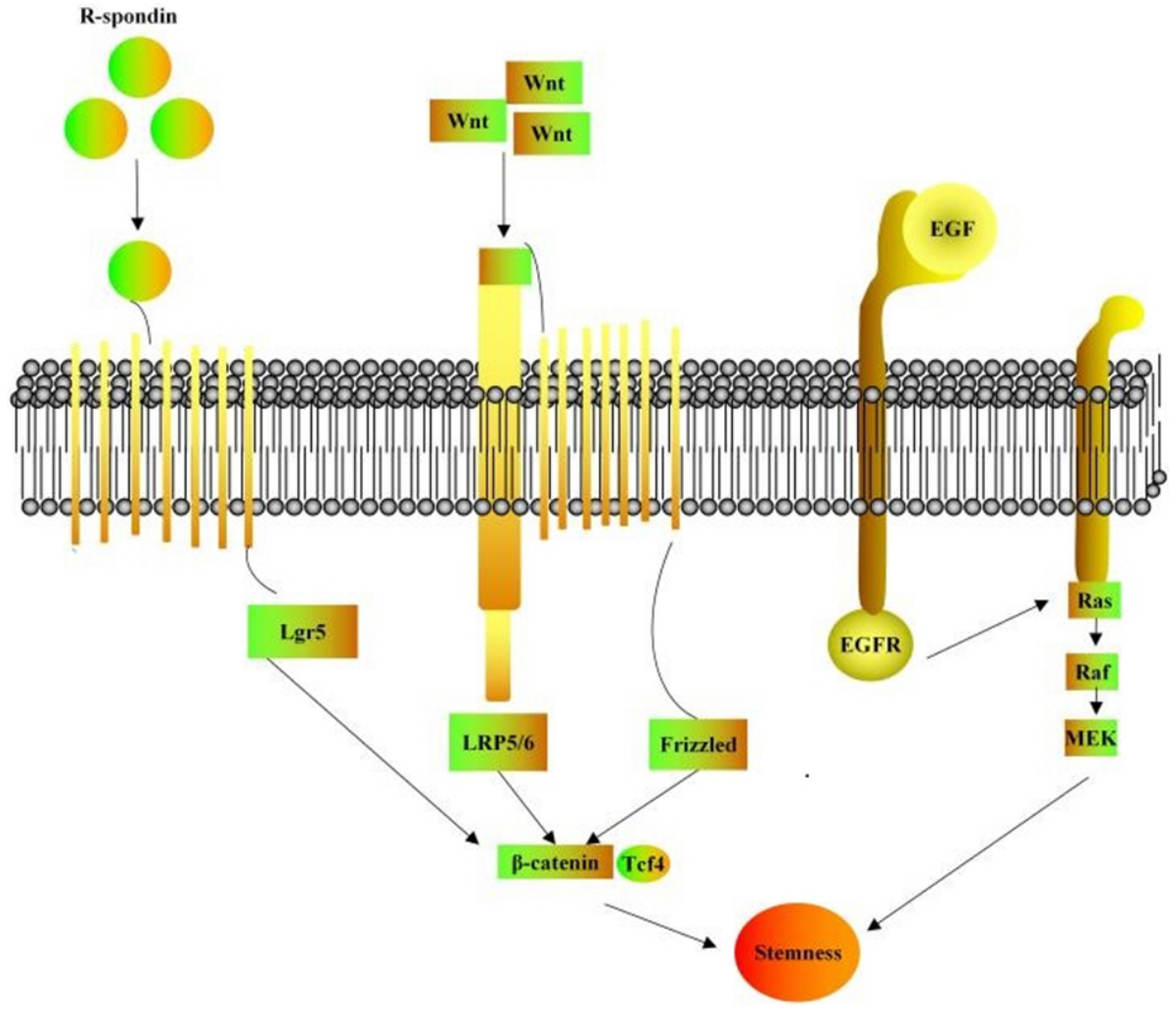

Fig. 2. The mechanism of R-Spondin, Wnt and EGF. 
noids and primary tumors are mutated. The similarity of characteristics and gene profile expression can reach $60 \%$ or more (28). Moreover, breast cancer organoids present all subtypes of solid tumors, which can be used as an effective in vitro model for this study's use of breast cancer organoids for high-throughput screening and personalized treatment, in combination with gene editing technology and the use of normal tissue-derived organoids for drug toxicity and side effects detection (Fig. 3).

\section{High-throughput drug screening and precision medicine}

The selection of tumor drugs and treatment options determines the prognosis of patients, and the heterogeneity of tumors determines the precision of individualized medicine to enhance the treatment effect for specific patients. Both the cell phenotype and tissue structure of breast cancer organoids can indicate the biological characteristics of solid tumors in patients. Moreover, as the three-dimensional culture system of breast cancer organoids has been built and optimized progressively, the organoid functions of batch culture can affect multiple drugs simultaneously. By screening, individualized treatment options can be quickly and accurately obtained. In contrast to the two-dimensional planar cell culture system of breast cancer organoids, the assessed results of the drug efficacy are closer to the actual effect. The results of the sensitivity of different clinical drug regimens can be visually presented. Several studies reported the assessed sensitivity up to $100 \%$, the specificity of $93 \%$, and the positive rate of $88 \%$ (31). Cassidy et al. (32) used breast cancer organs to screen out 18 anticancer drugs; they found that the in- hibition rate of pemetrexed combined with carboplatin, gemcitabine and pirlotinib was $60 \%$ more than those of other regimens. When this method is used in specific patients, the efficacy will be higher than the recommended standard treatment. The breast cancer organoids produced by Her2 over-expressed tissues are susceptible to tamoxifen. Under the concentration of the drug over $1 \mu \mathrm{m}$, the breast can be affected. The killing effect of cancer cells reaches over $50 \%$, complying with the results presented in patients (13). Breast cancer-like organoids are capable of achieving high-throughput screening of different anticancer drugs, and the specific treatment schemes that are more suitable for patients are taken from existing treatment schemes or emerging schemes, indicating the clinical application value of organoids in precision medicine.

\section{Breast cancer gene profile based personalized therapy}

Gene mutations critically lead to the formation of breast cancer, and different patients, different pathological types and even the identical disease type show differences in gene expression. Besides, targeted drugs targeting different mutation subtypes are vital to treatment. In the cytokine-added medium, stem cells can exhibit other genotypes if the original tissues are not differentiated. Nearly $90 \%$ of biopsies of one patient contain all the differentiated genotypes. Thus, it is of high significance value to study the association between breast cancer tissue genotype and anticancer drugs in vitro with organoid models (2). The model is capable of vertifying genetic variations in cancer progression and identifying and elucidating cancer progression genes, treatment responses, and outcomes associated with early to late stages. Accordingly, the orga-

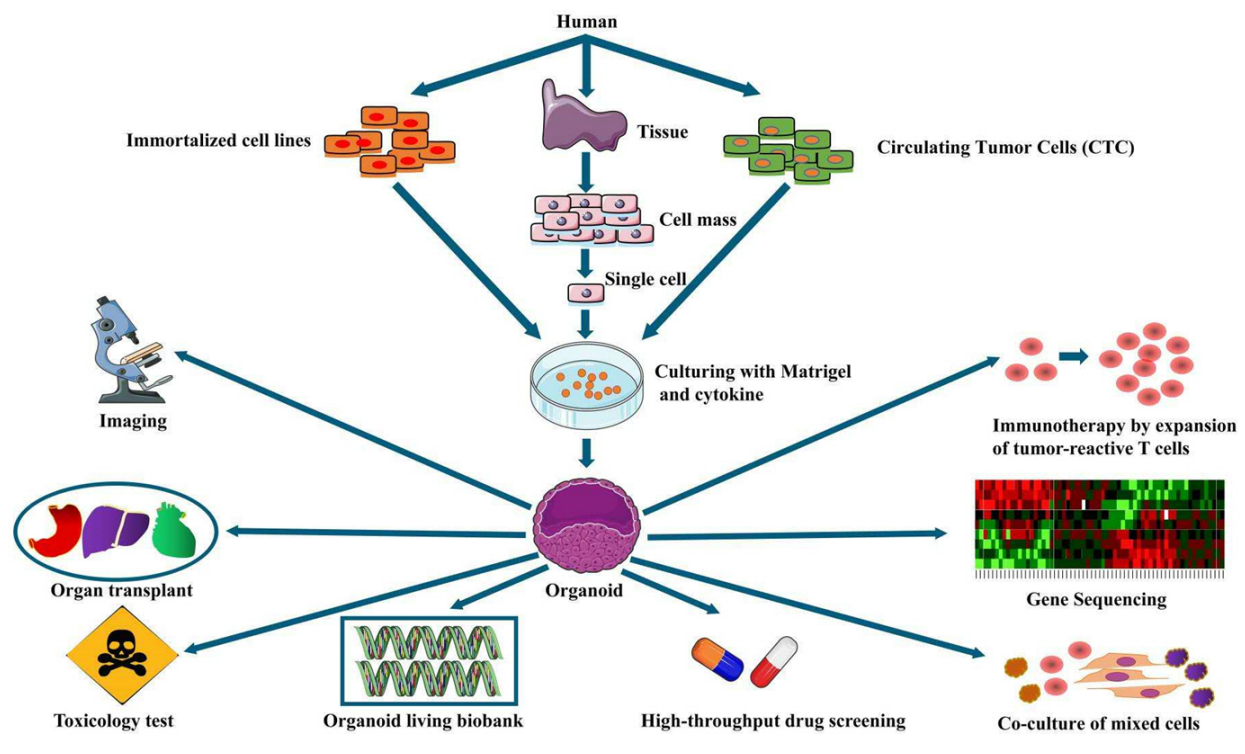

Fig. 3. The applications of organoids. 
noid model is used to explore the breast cancer tissue genotype and resistance, the relevance of cancer drugs is high. The proposed gene-drug combination complies with the tumor's abnormal genome to select the appropriate therapeutic drug. Unlike broad-spectrum anticancer drugs, patient tissue-derived organoids can be adopted to reproduce various mutant genes, and drugs can be developed and applied in a targeted manner. To fulfill the purpose of personalized treatment, Friedman et al. (33) performed genetic tests on breast cancer-like organs in specific patient; they found many mutated genes different from the source tissues. A novel poly (ADP-ribose) polymerase inhibitor was employed. (PARPis) specifically inhibits DNA damage and repair of breast cancer tumor cells, and then the purpose of personalized treatment for such breast cancer patients was achieved. With WGS sequencing method to find patients with high BRCAl/2 expression (e.g., PARP is), olaparib and Nilapali are more sensitive. Thus, breast cancer organoid genotypes are associated with drugs, which also enriches treatment options while achieving targeted treatments (34).

\section{Toxicity testing}

Besides the use of breast cancer organs for high-throughput screening of drugs and personalized treatment based on breast cancer gene profiles, it has also been extensively adopted in toxicology -under the development of novel drugs. New drugs developed for breast cancer are commonly tested for their effects and safety on animal models. In the case of little effect of drugs, they are usually treated by expanding the types or doses of anticancer drugs. Accordingly, drug accumulation often adversely affects patients' livers. Kidney function generates 'toxic side effects. Weeber et al. (35) exploited- breast cancer tissue-derived organoids to continuously controlthe dose and compatibility of various drugs before novel drugs were adopted to treat breast cancer patients. Such application solves the excessive abuse and limitation of animal models in the development of novel drugs, which - shortens the time required for drug detection to satisfy the standards and also creates ideas to deal with excessive anticancer drugs in clinical treatment.

\section{Immunotherapy and organoids transplant}

After the initial success of immunotherapy in melanoma, researchers in oncology have shifted their emphasis to their respective research directions. Co-culture of tumor organoids are derived from colorectal cancer (CRC) and non-small cell lung cancer (NSCLC) and peripheral blood lymphocytes of corresponding patients. After two tweeks of co-cultivation, the tumor organoids are recognized, presented and then processed -with the immune system as new organisms,; Subsequently, the patient's immune response is started. $50 \%$ of autologous tumor organoids enrich the $\mathrm{CD} 8+\mathrm{T}$ natural killer cells in the peripheral blood of patients, as well as promoting the secretion of IFN $\gamma$ in CD8 $+\mathrm{T}$ cells and the up-regulation of CD107a. Besides, the recruited $\mathrm{T}$ cells are capable of specifically acting on tumor-like organoids without targeting other nomal tissues of the patient under no - killing -reaction.; Tumor-like organoids decrease in diameter and are accompanied by extensive apotosis. This exhibits great advantages for the discovery of CRC and NSCLC primarily employed for immunotherapy of tumors derived from epithelial tissues. Moreover, it provides a reference for immunotherapy of breast cancer organoids derived fromepithelial cells (36). In addition, normal breast tissues from breast cancer patients are adopted for in vitro organoid culture, and the formed mammary gland organoids are re-transplanted into the patients receiving mastectomy, as autologous transplantation, immune rejection will not occur. Therefore, this technology is more conducive to organ transplantation, showing high development potential.

\section{Challenges and Future Directions}

The in vitro culture of breast cancer organoids creates the optimal model for tumor biology at the current stage, and its application in the screening of novel tumor drugs has significantly elevated the rate of clinical transformation. At present, the organoids generally take $4 \sim 6$ weeks from cultivation to testing, which are used to evaluate clinical efficacy. For patients who are urgent need of treatment, acquiring to the relating consequences of high-throughput screening of drugs, doctors are usually followed to guidelines and empirical plans, which leads to excessive therapies. Though breast cancer organoids can better reflect the patient's disease situation, we also note that when the culture system is being built, complete technology is still lacked to simultaneously connect both organoids and tumor microenvironment (37). Heterogeneity remains different from solid tumors. Moreover, More critical aspects affecting yield include whether the source tissue is healthy or tumor tissue, as well as the number of stem cells obtained after enzyme digestion. Healthy tissue organoids achieve the success rate of nearly $20.83 \%$, while that of cancer tissue reaches $87.5 \%$ (5). at present, the breast cancer-like organs that we cultivate from patients are primarily surgical tissue or puncture, whereas the tissues in different locations cover different numbers of breast cancer 
cells, and some tissues are liquefied layer and over-differentiated from the outside to the inside layer, tumor cell layer, and necrotic layer in the center of the tumor, in the sampling process, it is difficult to ensure that all are tumor tissues, and the number of cells obtained after enzyme digestion is different, which affects the survival rate of organoid culture. Moreover, matrigel and cytokines have different concentrations in different laboratories, which is not conducive to a unified evaluation of the formation rate of breast cancer organ cultures (38-40). To be specific, it lacks mesenchymal cells, so it has no nerve and vascular system, and it has great differences with solid tumors in constituting the tumor microenvironment.

With the advent of advanced imaging technologies (e.g., optical metabolic imaging (OMI)), the time of using organoids to determine the effectiveness of treatment options can be reduced to $72 \mathrm{~h}$, and the invalid scheme of organoids can be more accurately identified, so as to avoid patients having received excessive treatment (41).

In response to the problem of low cell content in tumor tissue, we can use multi-point biopsy. For experimental differences caused by different product batches and inconsistent schemes in the culture system, we can adjust matrigel by adding collagen protein composition, so the same substrate is applied in independent experiments to ensure the repeatability of the experiment (14). Moreover, Besides, recent studies have continuously pointed out that we can solve the shortcomings of single organoid system components by adding other cells to form a "co-culture" system. Dijkstra et al. (36) employed the patient's tumor-like organs to activate T lymphocytes to amplify the patient's immune function. The results of their experiment provide novel insights into the study on breast cancer immunotherapy. Moreover, the continuous advancement of microfluidic technology and new equipment will further expand the application scenarios and enhance the efficiency of tumor organoids.

\section{Discussion}

In the conventional cell models, oxygen, nutrients, metabolities and signaling molecules can be used infinitely, however, the cancer cells in the body have limited utilization of various substances due to the three-dimensional structure of the tumor mass, consequently, causing various degrees of influence on the expression of cancer cell molecules and biological behaviors. Besides, patient-derived xenografts cannot be screened for high-throughput of drugs. Compared with the above models, organoids are easy to be cultured, and involve the advantages of cell models and
PDX. Therefore, it has become the first choice for emerging breast cancer in a short time of research models in vitro.

Although breast cancer organoids are widely used, there are still apparent deficiencies, such as: lack of mesenchymal cells, nerves, blood vessels, and immune system. The model that is constituted by single breast cancer is still quite different from the solid tumor in terms of tumor heterogeneity. The relating studies have continuously pointed out that we can form a "co-culture" system by adding corresponding cell components to restore the "tumor microenvironment" to a certain extent, for instance: lymphocytes andfibroblasts, to achieve the purpose of highly reproducing solid tumors in vitro.

Breast cancer organoids can not only explore the role of tumor stem cells, so that we can study the occurrence, development and driving factors of tumors from normal cells, invasive cancers to metastatic cancers; but used to the chemotherapy, combined with gene editing technology to interfere with the progress of breast cancer, assessment of drug toxicity and immunotherapy autologous transplantation. Organoids can be generated from surgical samples, as well as from endoscopic fine needle aspiration samples. Thus, the model is capable of summarizing various disease stages and clinical conditions. Moreover, organoids can be generated from a small amount of tissue and completed efficiently, which can be employed for drug testing and assessment of potential diagnostic biomarkers; they can also verify multiple drivers of tumor progression in vitro. Overall, it is considered that as fueled by the advancement of technology and research in relevant fields, the application of breast cancer organs will be increasingly widespread, bringing invaluable value to more clinical treatment and academic study.

\section{Potential Conflict of Interest}

The authors have no conflicting financial interest.

\section{Author Contributions}

Conceptualization: Jin Yu, Wei Huang

Investigation: Jin $\mathrm{Yu}$

Resources: Jin $\mathrm{Yu}$

Supervision: Jin $\mathrm{Yu}$

Writing - original draft: Jin Yu, Wei Huang

Writing - review \& editing: Jin $\mathrm{Yu}$

\section{References}

1. Siegel RL, Miller KD, Jemal A. Cancer statistics, 2019. CA Cancer J Clin 2019;69:7-34 
2. Chen KY, Srinivasan T, Lin C, Tung KL, Gao Z, Hsu DS, Lipkin SM, Shen X. Single-cell transcriptomics reveals heterogeneity and drug response of human colorectal cancer organoids. Conf Proc IEEE Eng Med Biol Soc 2018;2018: 2378-2381

3. Moreira L, Bakir B, Chatterji P, Dantes Z, Reichert M, Rustgi AK. Pancreas 3D organoids: current and future aspects as a research platform for personalized medicine in pancreatic cancer. Cell Mol Gastroenterol Hepatol 2017;5: 289-298

4. Broutier L, Mastrogiovanni G, Verstegen MM, Francies HE, Gavarró LM, Bradshaw CR, Allen GE, Arnes-Benito R, Sidorova O, Gaspersz MP, Georgakopoulos N, Koo BK, Dietmann S, Davies SE, Praseedom RK, Lieshout R, IJzermans JNM, Wigmore SJ, Saeb-Parsy K, Garnett MJ, van der Laan LJ, Huch M. Human primary liver cancerderived organoid cultures for disease modeling and drug screening. Nat Med 2017;23:1424-1435

5. Risbridger GP, Toivanen R, Taylor RA. Preclinical models of prostate cancer: patient-derived xenografts, organoids, and other explant models. Cold Spring Harb Perspect Med 2018;8:a030536

6. Mazzucchelli S, Piccotti F, Allevi R, Truffi M, Sorrentino L, Russo L, Agozzino M, Signati L, Bonizzi A, Villani L, Corsi F. Establishment and morphological characterization of patient-derived organoids from breast cancer. Biol Proced Online 2019;21:12

7. Wilson HV. A new method by which sponges may be artificially reared. Science 1907;25:912-915

8. Emerman JT, Pitelka DR. Maintenance and induction of morphological differentiation in dissociated mammary epithelium on floating collagen membranes. In Vitro 1977;13: 316-328

9. Petersen OW, Rønnov-Jessen L, Howlett AR, Bissell MJ. Interaction with basement membrane serves to rapidly distinguish growth and differentiation pattern of normal and malignant human breast epithelial cells. Proc Natl Acad Sci U S A 1992;89:9064-9068

10. Fata JE, Mori H, Ewald AJ, Zhang H, Yao E, Werb Z, Bissell MJ. The MAPK (ERK-1,2) pathway integrates distinct and antagonistic signals from TGFalpha and FGF7 in morphogenesis of mouse mammary epithelium. Dev Biol 2007;306:193-207

11. Sato T, Vries RG, Snippert HJ, van de Wetering M, Barker N, Stange DE, van Es JH, Abo A, Kujala P, Peters PJ, Clevers H. Single Lgr5 stem cells build crypt-villus structures in vitro without a mesenchymal niche. Nature 2009; 459:262-265

12. de Visser KE, Ciampricotti M, Michalak EM, Tan DW, Speksnijder EN, Hau CS, Clevers H, Barker N, Jonkers J. Developmental stage-specific contribution of $\mathrm{LGR}^{+}$cells to basal and luminal epithelial lineages in the postnatal mammary gland. J Pathol 2012;228:300-309

13. Sachs N, de Ligt J, Kopper O, Gogola E, Bounova G, Weeber F, Balgobind AV, Wind K, Gracanin A, Begthel H, Korving J, van Boxtel R, Duarte AA, Lelieveld D, van
Hoeck A, Ernst RF, Blokzijl F, Nijman IJ, Hoogstraat M, van de Ven M, Egan DA, Zinzalla V, Moll J, Boj SF, Voest EE, Wessels L, van Diest PJ, Rottenberg S, Vries RGJ, Cuppen E, Clevers H. A living biobank of breast cancer organoids captures disease heterogeneity. Cell 2018;172:373386.e10

14. Tuveson D, Clevers H. Cancer modeling meets human organoid technology. Science 2019;364:952-955

15. Cariati M, Naderi A, Brown JP, Smalley MJ, Pinder SE, Caldas $\mathrm{C}$, Purushotham AD. Alpha-6 integrin is necessary for the tumourigenicity of a stem cell-like subpopulation within the MCF7 breast cancer cell line. Int J Cancer 2008; 122:298-304

16. Yousefnia S, Ghaedi K, Seyed Forootan F, Nasr Esfahani $\mathrm{MH}$. Characterization of the stemness potency of mammospheres isolated from the breast cancer cell lines. Tumour Biol 2019;41:1010428319869101

17. Yu M, Bardia A, Aceto N, Bersani F, Madden MW, Donaldson MC, Desai R, Zhu H, Comaills V, Zheng Z, Wittner BS, Stojanov P, Brachtel E, Sgroi D, Kapur R, Shioda T, Ting DT, Ramaswamy S, Getz G, Iafrate AJ, Benes C, Toner M, Maheswaran S, Haber DA. Cancer therapy. Ex vivo culture of circulating breast tumor cells for individualized testing of drug susceptibility. Science 2014;345:216-220

18. Grimshaw MJ, Cooper L, Papazisis K, Coleman JA, Bohnenkamp HR, Chiapero-Stanke L, Taylor-Papadimitriou J, Burchell JM. Mammosphere culture of metastatic breast cancer cells enriches for tumorigenic breast cancer cells. Breast Cancer Res 2008;10:R52

19. Hanahan D, Weinberg RA. The hallmarks of cancer. Cell 2000;100:57-70

20. Koledova Z. 3D coculture of mammary organoids with fibrospheres: a model for studying epithelial-stromal interactions during mammary branching morphogenesis. Methods Mol Biol 2017;1612:107-124

21. Kuperwasser C, Chavarria T, Wu M, Magrane G, Gray JW, Carey L, Richardson A, Weinberg RA. Reconstruction of functionally normal and malignant human breast tissues in mice. Proc Natl Acad Sci U S A 2004;101:4966-4971

22. Rios AC, Clevers H. Imaging organoids: a bright future ahead. Nat Methods 2018;15:24-26

23. Xu H, Lyu X, Yi M, Zhao W, Song Y, Wu K. Organoid technology and applications in cancer research. J Hematol Oncol 2018;11:116

24. Sato T, Clevers H. Growing self-organizing mini-guts from a single intestinal stem cell: mechanism and applications. Science 2013;340:1190-1194

25. Dontu G, Abdallah WM, Foley JM, Jackson KW, Clarke MF, Kawamura MJ, Wicha MS. In vitro propagation and transcriptional profiling of human mammary stem/progenitor cells. Genes Dev 2003;17:1253-1270

26. Sato T, Clevers H. SnapShot: growing organoids from stem cells. Cell 2015;161:1700-1700.el

27. Huang SX, Islam MN, O'Neill J, Hu Z, Yang YG, Chen YW, Mumau M, Green MD, Vunjak-Novakovic G, 
Bhattacharya J, Snoeck HW. Efficient generation of lung and airway epithelial cells from human pluripotent stem cells. Nat Biotechnol 2014;32:84-91

28. Jardé T, Lloyd-Lewis B, Thomas M, Kendrick H, Melchor L, Bougaret L, Watson PD, Ewan K, Smalley MJ, Dale TC. Wnt and Neuregulin1/ErbB signalling extends 3D culture of hormone responsive mammary organoids. Nat Commun 2016;7:13207

29. Wu Y, Tran T, Dwabe S, Sarkissyan M, Kim J, Nava M, Clayton S, Pietras R, Farias-Eisner R, Vadgama JV. A83-01 inhibits TGF- $\beta$-induced upregulation of Wnt3 and epithelial to mesenchymal transition in HER2-overexpressing breast cancer cells. Breast Cancer Res Treat 2017;163:449460

30. Schwartz M, Böckmann S, Borchert P, Hinz B. SB202190 inhibits endothelial cell apoptosis via induction of autophagy and heme oxygenase-1. Oncotarget 2018;9:23149-23163

31. Vlachogiannis G, Hedayat S, Vatsiou A, Jamin Y, FernándezMateos J, Khan K, Lampis A, Eason K, Huntingford I, Burke R, Rata M, Koh DM, Tunariu N, Collins D, Hulkki-Wilson S, Ragulan C, Spiteri I, Moorcraft SY, Chau I, Rao S, Watkins D, Fotiadis N, Bali M, DarvishDamavandi M, Lote H, Eltahir Z, Smyth EC, Begum R, Clarke PA, Hahne JC, Dowsett M, de Bono J, Workman P, Sadanandam A, Fassan M, Sansom OJ, Eccles S, Starling N, Braconi C, Sottoriva A, Robinson SP, Cunningham D, Valeri N. Patient-derived organoids model treatment response of metastatic gastrointestinal cancers. Science 2018; 359:920-926

32. Cassidy JW, Batra AS, Greenwood W, Bruna A. Patient-derived tumour xenografts for breast cancer drug discovery. Endocr Relat Cancer 2016;23:T259-T270

33. Friedman AA, Letai A, Fisher DE, Flaherty KT. Precision medicine for cancer with next-generation functional diagnostics. Nat Rev Cancer 2015;15:747-756
34. Shi Y, Inoue H, Wu JC, Yamanaka S. Induced pluripotent stem cell technology: a decade of progress. Nat Rev Drug Discov 2017;16:115-130

35. Weeber F, Ooft SN, Dijkstra KK, Voest EE. Tumor organoids as a pre-clinical cancer model for drug discovery. Cell Chem Biol 2017;24:1092-1100

36. Dijkstra KK, Cattaneo CM, Weeber F, Chalabi M, van de Haar J, Fanchi LF, Slagter M, van der Velden DL, Kaing S, Kelderman S, van Rooij N, van Leerdam ME, Depla A, Smit EF, Hartemink KJ, de Groot R, Wolkers MC, Sachs N, Snaebjornsson P, Monkhorst K, Haanen J, Clevers H, Schumacher TN, Voest EE. Generation of tumor-reactive T cells by co-culture of peripheral blood lymphocytes and tumor organoids. Cell 2018;174:1586-1598.e12

37. Fernández-Periáñez R, Molina-Privado I, Rojo F, GuijarroMuñoz I, Alonso-Camino V, Zazo S, Compte M, AlvarezCienfuegos A, Cuesta AM, Sánchez-Martín D, AlvarezMéndez AM, Sanz L, Alvarez-Vallina L. Basement membrane-rich organoids with functional human blood vessels are permissive niches for human breast cancer metastasis. PLoS One 2013;8:e72957

38. Weigelt B, Ghajar CM, Bissell MJ. The need for complex 3D culture models to unravel novel pathways and identify accurate biomarkers in breast cancer. Adv Drug Deliv Rev 2014;69-70:42-51

39. Lee GY, Kenny PA, Lee EH, Bissell MJ. Three-dimensional culture models of normal and malignant breast epithelial cells. Nat Methods 2007;4:359-365

40. Huch M, Knoblich JA, Lutolf MP, Martinez-Arias A. The hope and the hype of organoid research. Development 2017; 144:938-941

41. Walsh AJ, Cook RS, Skala MC. Functional optical imaging of primary human tumor organoids: development of a personalized drug screen. J Nucl Med 2017;58:1367-1372 